\title{
Schizophrenia and conscious goal selection: Bodily representation and free energy imperatives
}

\author{
Denis Larrivee* \\ Loyola University Chicago, 1032 W Sheridan Rd, Chicago, IL, USA
}

\begin{abstract}
The implementation of bodily representation in dynamical motor performance constitutes strong evidence of the need to frame the self in the context of the body. Conversely, the variation in consciously perceived self-constructs, elicited in different interactive contexts, implicate additional physical determinants that shape the representational content of the self. Recent proposals suggest that probabilistic inferences of energy consumption modify conscious and intentional behavior, a mechanism which may impact the self- representation. The inability of schizophrenia patients to attribute intentional actions to the self-representation, but not the failure of automatic processes to identify self-generated motions, however, suggests that these determinants are unlikely to involve energy inferencing, which appears to influence unconscious and automatic action identification.
\end{abstract}

\section{Introduction}

As a consciously sensed construct the self is variably perceived [1]. In circumstances of action attribution, the self-functions as a source from which bodily actions emerge, in self/other interactions, as a social agent that is distinguished from the other, and in the conscious awareness of thought and experience, as a spatiotemporal reality that knows itself, in John Locke's words, in different times and places.

Nonetheless, a key and consistent relationship in varied selfrepresentations is the association of the self with a representation of the whole body, which is physically realized in the neural activity of the brain. Disturbances of self that traditionally mark diagnostic evaluation of the schizophrenia patient, for example, typically include an abnormal sense of body ownership, loss of ego boundary, or a confused sense of action attribution, each of which entail bodily associations [2-4]. Furthermore, infants perseverating toward a hidden object where they have previously reached for it, rather than where they have last seen it hidden, is viewed as a failure of one of a class of executive control tasks, an inability explained by yet to develop mechanisms needed for situating the motor plan with respect to a representation of the self as the whole body [5].

Accordingly, the sense of self entails implicit and tacit feelings of body relationship. Expressed through body ownership it is evocative of the body as both the physical reality of the self and as the personal possession of the self. It can occur, therefore, whether bodily actions are self-initiated, or whether they are actions done to the body [6]. In action attribution, for instance, the perceptual realization that the body is the source of its actions links the self-percept to bodily action undertaken for the good of the whole individual. In still another case the body serves to configure the spatial domain occupied by the self to distinguish it from the other in social interactions [7].

This common realization of the body in varying dynamical contexts constitutes strong evidence of the need to frame the self as a bodily representation. Philosophically, the need to instantiate the self within the framework of the body can be understood as an a priori condition required for autonomous existence. Goal directed actions, for example, acquire intelligibility only if performed to secure an end for the whole individual. Accordingly, actions undertaken for the good of the whole must define it as an objective physical reality for which the actions bear significance. The process of appraisal then configures the manner by which the relationship between self and goal can be articulated. Within a thermodynamic context, for example, discrete actions clearly have repercussions for the energy needs of the entire body [8].

On the other hand, the variation in consciously perceived selfconstructs, elicited in different interactive contexts, implicate additional physical determinants that shape the representational content of the self as a function of consciously sensed experiential exigencies. As a corollary, psychiatric diseases impacting the self can be expected to variably impact the capacity to link the representation of the body to the representational content of the self. Indeed, action attribution, but not automatic processing of bodily actions is deficient in schizophrenia patients [9]. This observation, notably, led Jeannerod [9] to conclude that in action attribution there exist two levels of self-recognition, one automatic and unconsciously employed for action identification, and a second, deliberative and conscious that additionally incorporates intentional desires. That is, the disease's etiology involves an inability to attribute intentional actions to the self-representation, but not a failure of automatic processes that identify self-generated motions.

In at least one proposal the consciously evoked self-construct entails six different representations that are assembled in successive developmental periods [1]. In this proposal a topologically defined and subsequently invariant spatial representation is assembled first in

*Correspondence to: Denis Larrivee, Loyola University Chicago, 1032 W Sheridan Rd, Chicago, IL, USA, Tel: +1 773-274-3000; E-mail: sallar1@aol.com

Key words: schizophrenia; probabilistic; energy inferencing

Received: October 30, 2018; Accepted: November 20, 2018; Published: November 23, 2018 
infants, that is, a bodily representation that is delimited by the body's physical structure. With development, successive self-representations overlay the representational content of this proto self. Hence, the proposal predicts that self-representations will share overlapping brain regions with those assembled earlier in development. On the other hand, recent observations of neural activity associated with body ownership occupy distinct as well as common brain regions with activity linked to the peri-personal space [10]. These latter observations instead appear to reflect a model in which the perceptual construct is elicited uniquely in the context of differing goal-oriented activities. Together, these observations appear to link functional and goal-oriented distinctions to the representation of the body but leave unresolved the significant distinctions between conscious and unconscious self-imagery and how this distinction may relate to the articulation of varying intentional goals in which the body is participatory. Significantly, among determinants likely to influence this articulation are factors constraining the attainment of goals, particularly energy use.

Emphasis on energy as a behavioral modifier has focused on linking intentional activity to constraints imposed on the perception of their likely outcome, that is, the making of inferences that will minimize energy consumption $[11,12]$. This paper will consider the influence of energy inferencing as a factor affecting the desires and intentions of consciously initiated, goal directed activity. The paper will argue that energy inferencing is likely to significantly influence automatic and unconscious action identification, but is unlikely to affect intentional and conscious ones, a conclusion obtained from schizophrenia observations.

\section{The motor image in dynamical planning}

The conception of the neural representation of the self, evoked in circumstances where the body is dynamically engaged, can be traced to the evolution in understanding of the motor image [13]. In its current conception the image constitutes a covert action undertaken only mentally and as a simulation of a non-executed action. That is, the motor image contains the feature elements of a motor trajectory and so contains the projected series of motions that are prospective for execution.

Insights drawn from the motor image reveal that bodily representation is a key feature that frames the elements of the plan as teleologically oriented; that is, one that inscribes actions linking an agent with an objective destination. So inscribed, actions are thereby executed as a coherent and coordinated dynamical ensemble, which have a causal origin linked to the whole individual. Accordingly, features of the motor plan entail mutual though distinct contributions from peripheral as well as central origins, underscoring the essential unity of dynamic performance even in its covert formulation.

Central influences can be demonstrated by the presence of movements undertaken following dorsal root deafferentation [14]. When the original relationship between the movement and its location in space is altered, limb movements fail to correspond to their expected trajectory, instead missing their targets. This mis reaching has been shown to be due to their dependence on previously stored commands sent to the sensory cortex $[15,16]$ that references the original locations as the spatial origins of the movements; which is also to say that stored central influences govern the specific motor output that leads to the wrong limb positioning.

Sensory cues, on the other hand, are required in continuous motions in order to correctly respond to the ongoing changes in limb and bodily position that occur during movements. This peripheral sensorial contribution continually updates information of the body's spatial locations enabling adjustment in motor trajectories. In effect, the functional synergy between peripheral sensorial and central motor influence creates a perception-action loop that regulates the perception of the external world and the individual's interaction with it [17]. Their significance has been demonstrated in many studies including those investigating the body's perceptual and dynamical interaction with the environment in developing infants, acquired habitual motor abilities, biodynamic studies showing that movement and proprioception are intrinsically related to perception, and the motoric encoding of actions like reaching [18-20].

Crucially, the body's topological 3-dimensional configuration is essential to coordinated performance. By siting sensory input to the body's perimeter, it is intuitive that information flow can be modulated as a function of the body's configuration [21]. Existing studies now show that the assembly of the body image grounds these motions to a dynamic whole from which individual bodily actions emerge, and that somatotopic input is fundamental to the construction of the image. Beginning with such afferent input a three-dimensional postural image is successively built up from segmented zones that are progressively and hierarchically assembled enroute to the brain, with much of the assembly apparently occurring enroute at spinal and brain stem levels. In effect the experiential and dynamical operation of this recurrent framework structures a prototypical platform where peripherally enacted and complex integration increasingly occurs. Interactive events with the body are thereby mapped to their respective bodily locus yielding a temporal evolution of bodily contextualized events. Building on this platform, neural activity can be assimilated from their various sittings to generate a common dynamical representation, that is, a bodily entity of which the representation is indicative [22].

\section{Linking bodily representation, energy consumption, and action identification}

In the absence of the whole image, actions are not undertaken [5] apparently because they lack holistic significance. Hence, the body's representation appears necessary to confer unity for performance or other ends. Crucially, this has implications for physicochemical demands of bodily action, that is, how the self-accommodates energy constraints to limit interaction with a constantly changing suite of daily circumstances.

Because all organisms exist far from energy equilibrium, there is an ongoing need to acquire free energy from the environment to sustain motile activity and bodily metabolism. The need to acquire energy for the whole organism and to acquire it from sources beyond the organism, dictate dual tracks in organismal development [23]; that is, dual capacities that are subject to separate evolutionary molding yet intrinsically depend on one another. To secure free energy that can replenish energy dissipating, recursive remodeling, interactivity with the external environment, accordingly, must be coordinated with an internal metabolic order. The need for this coordination thus places constraints on the organismal organizational order creating a system wide suite of interdependencies.

Situated by the need to interact with the environment the self mobilizes the internal organizational order to seek out and replenish depleted reserves needed to maintain organismal independence. Living organisms, in fact, are uniquely characterized by their autonomy [24]; that is, an organized capacity to acquire free energy from the external 
environment to direct its use to their own ends. Such ends are widely variable, motivated by a spectrum of goals beyond those dictated by energy acquisition alone, and articulated by the self, which confers unity in their autonomous pursuit [25].

Significantly, because energy use is necessarily immanent and holistic, viability is critically dependent on maximizing energy efficiency. In a topologically closed system this means that there is a direct link between organismal order and energy expenditure, which thus acts as a constraint on behavioral range; that is, it is the system wide requirement for maximal energy efficiency that imposes limitations on the sorts of behaviors that may be undertaken by the individual.

In recognition of this holistic constraint, several influential theories propose that inscribed in the motor planning trajectories are causal probabilistic inferences about the likely interactive scenarios and the relative energy use incurred in performance [26]. In other words, within the representational content of the motor image there are neural constraints permitting only a certain range of behavioral outcomes in order to minimize the use of energy; accordingly, these constitute regulatory mechanisms for maximizing body-wide energy efficiency. Indeed, these cognitive structures function to track the state of the body and encode it [27]. As an ongoing physico-chemical imperative energy inferences influence the evolutionary legacy of interactive behavior.

Given their evolutionary legacy these possibilities can be expected to occupy a phenomenal domain that is immediate and responsive, without need for recourse to conscious evaluation. This is to say that unlike intentional actions they can be immediately and unconsciously evoked for most responses needed for action sequences. Consistent with this interpretation, schizophrenic patients, for whom the sense of self agency is pathologically affected, resemble healthy subjects in automatically adapting their movements to visuomotor conflicts to reach a visual target, despite distortions in feedback [28]. However, such patients fare much worse than normal individuals in tests requiring the use of conscious agency for judgments [7]. These individuals, therefore, appear able to automatically undertake actions that maximize energy efficiency for the whole body, but are unable to contextualize conscious actions to the self.

\section{Schizophrenia and conscious actions: the body image and free energy imperatives}

Taken together, the inability of schizophrenia patients to consciously attribute bodily actions to the self-reveals a failure to link the intentions and desires of a protagonist to an intact representation of the body. Conversely, this means that during conscious actions normal individuals inscribe not only the bodily representation in the representational content of the self but also intentional information that is related to the goal being sought. Jeannerod [9] specifically identifies within this content the intentions, plans, and desires of the author of these actions. Accordingly, the feature elements of the conscious motor plan are no longer teleologically situated in these patients nor is the agent linked with an objective destination.

One outcome predicted by this understanding is that the representational content of the self-varies as a function of the specific goals consciously embraced by the author of those intentions. As a corollary, schizophrenia patients ought to be deficient in inscribing goal specific features within the self-representation; that is, the representation of the self in schizophrenia patients will not only differ with respect to the self-content of normal individuals but also with respect to the self-content contained in different goal contexts. This prediction is briefly considered here in two cases of willed actions that substantially differ with respect to the nature of the goal for which the actions are willed, self/other social interactions and action attribution.

As a class, social interactions are unique for their requirement to carefully delineate the self from the subjective features that characterize other individuals $[29,30]$. Indeed, as social 'animals' humans are especially oriented to self-other interactions. Research from social psychology indicates that the self-representation is not only unique but also shares common features that assist in identifying others like himself. Developmental processes for self-other perceptions, for example, entail two stages, one in which the self-percept is configured first through its association with the body, and a second in which the self-construct is elicited by intersubjective interactions [31]. Indeed, social neuroscience has revealed a broad cluster of dedicated cells, circuits, and zones that assist in distinguishing self from other that have been classed under theory of mind [30].

As a proxy for self-other interactions such distinctions have been related to an innate ability to recognize a spatial domain surrounding the body as the space inhabited by the self [7]. While this perception that the self-occupies a given location is amenable to experimental manipulation, entailing shifts in the perception of the location of self, such displacement has a finite range beyond which the self is no longer present. This limitation appears to relate to the need to associate the space with the body, which is needed to configure it. Significantly, the ability to localize the inhabited space of the self is substantially altered in subjects who have schizophrenia. In these individuals the self-disorder is characterized by a weak and shallow peri personal space between self and other, in which the self and other are not easily differentiated [32].

Goal directed actions that entail motor planning, such as those studied by Jeannerod [9], on the other hand, do not require the clear demarcation between self and other that are characteristic of social interactions. Instead, the bodily representation is modified to incorporate actions that have as their outcome an intended goal terminus; here, the author's intentions clearly differ from those evoked in social interaction.

Studies where the motor trajectory entails the intentional use of utensils [33], for example, show that self-mapping overlaps with the utensil revealing a coincidence between the goal and the intended motor trajectory, a coincidence not evidenced in schizophrenia patients [9].

The differences in the representational content of self in its conscious appropriation from that acquired unconsciously - or as a function of varying goal contexts - remain to be determined. Nonetheless, the selective influence of schizophrenia - and potentially that of other self-related psychiatric disorders [34] on the conscious appropriation of the self-implicates a fundamental distinction between conscious and unconscious realizations in the context of intentional goal directed actions. Since this does not appear to relate to the bodily representation per se - schizophrenia patients appear to possess an intact body representation, it is unlikely to impact inferencing mechanisms used for maximizing free energy efficiency (Figure 1) which necessarily relate to whole body dynamics.

Taken together, proposed energy inferencing mechanisms appear to inhibit motor plan formation that may exceed a criterion state during action identification, a process likely to occur in the premotor or posterior parietal cortex [9]. In conscious goal directed actions, on the other hand, the self representation appears to incorporate the body image together with the intentions and desires of the self, but these consciously evoked plans are not subject to energy inferencing constraints. Accordingly, this suggests the existence of a pathway that 


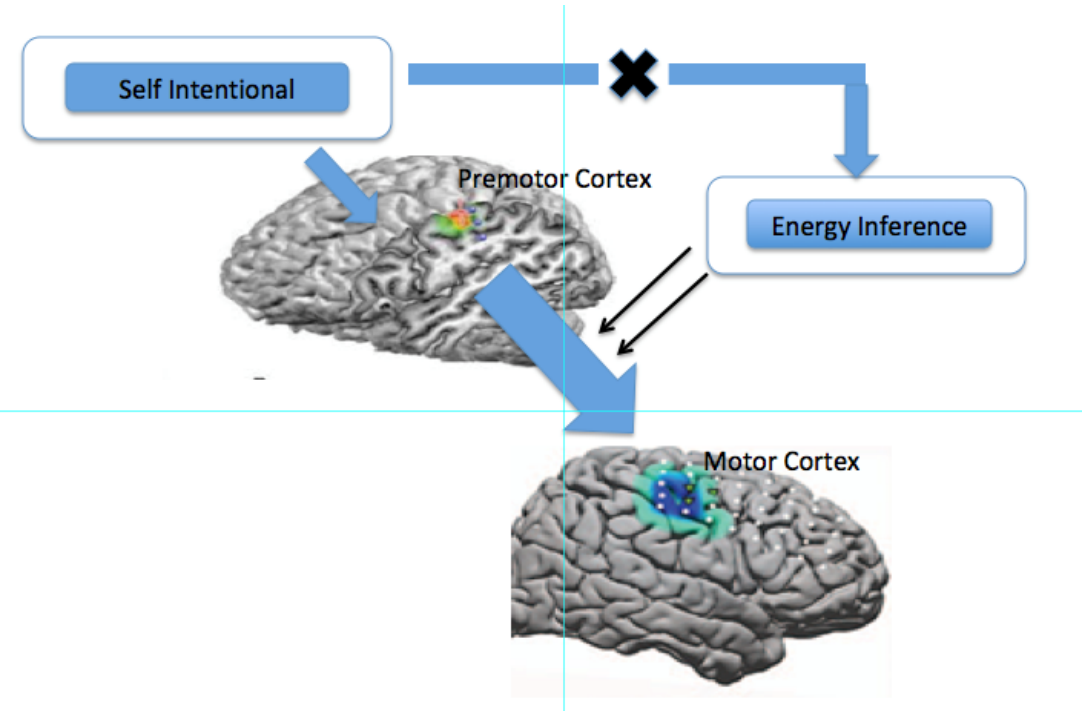

Figure 1. Lifting of energy inferencing constraints during conscious, intentional motor planning

inhibits constraints imposed by energy inferencing that functions to broaden the scope of conscious and intentional action.

In other words, such inferencing mechanisms require a tight coupling to the whole body in order to gauge potential energy use and are likely to involve multiple cell and circuit mechanisms, a coupling maintained in unconscious actions, but not in conscious, intentionally selected ones. Schizophrenia lesions, thus, reveal the existence of a class of actions for which the representational content of self is independent of constraints linked to energy assessment. They therefore suggest not only two distinct mechanisms associated with motor behavior, one unconscious for action identification and one conscious for intentional goals, but also the use of conscious behavior to limit constraints imposed by energy assessment.

\section{Conclusion}

A key and consistent relationship in varied self-representations is the association of the self with a representation of the whole body, realized through the neural activity of the brain. Consciously perceived self-constructs, however, display significant variation in representational content that appears related to intentional and goal directed outcomes. Schizophrenia lesions reveal that the latter are associated with a class of actions likely to be independent of constraints linked to energy assessment. Recent proposals suggesting the influence of energy inferencing on conscious and intentional behavior are unlikely to explain the variation in self construct and are more likely to be deployed unconsciously in a bodily assessment of action identification.

\section{References}

1. Riva G (2018) The neuroscience of body memory: From the self through the space to the others. Cortex 104: 241-260. [Crossref]

2. Blankenburg W (1971) The Loss of Natural Self-evidentness: A Contribution to the Psychopathology of Symptom-Deficient Schizophrenia. Ferdinand Enke, Stuttgart, Germany.

3. Schneider K (1959) Clinical Psychopathology. Grune \& Stratton, New York.

4. Ferri F, Frassinetti F, Mastrangelo F, Salone A, Ferro FM, et al. (2012) Bodily self and schizophrenia: the loss of implicit self-body knowledge. Conscious Cogn 21: 13651374. [Crossref]

5. Smith L (2009) Stability and flexibility in development. In: Spencer J, Thomas MSC, McClelland JL Toward a unified theory of development. Oxford University Press, Oxford.

6. Hur JW, Kwon JS, Lee TY, Park S (2014) The crisis of minimal self-awareness in schizophrenia: a meta-analytic review. Schizophr Res 152: 58-64. [Crossref]
7. Noel JP, Pfeiffer C, Blanke O, Serino A (2015) Peripersonal space as the space of the bodily self. Cognition 144: 49-57. [Crossref]

8. Grecos AP, Prigogine I (1972) Dissipative properties of quantum systems. Proc Natl Acad Sci U S A 69: 1629-1633. [Crossref]

9. Jeannerod M (2009) The sense of agency and its disturbances in schizophrenia: a reappraisal. Exp Brain Res 192: 527-532. [Crossref]

10. Grivaz P, Blanke O, Serino A (2017) Common and distinct brain regions processing multisensory bodily signals for peripersonal space and body ownership. NeuroImage 147: 602-618. [Crossref]

11. Rudrauf D, Bennequin D, Granic I, Landini G, Friston K, et al. (2017) A mathematical model of embodied consciousness. J Theor Biol 428: 106-131. [Crossref]

12. Friston K, Sengupta B, Auletta G (2014) Cognitive dynamics: from attractors to active inference. Proceedings IEEE 102: 427-445.

13. Jeannerod M (2005) Levels of representation of goal-directed actions. Oxford: Oxford University Press.

14. Lashley KS (1951) The problem of serial order in behavior. In: LA Jeffress, ed Cerebral mechanisms and behavior. New York: Wiley Press pp: 112-136.

15. Von Holst E, Mittelstaedt H (1950) The reafference principle. Interactions between central nervous system and periphery. Natural Sciences 37: 464-476.

16. Weigmann K (2013) Our sense of self. Phenomenology is a philosophical discipline that gives a detailed description of selfhood; it can contribute to understanding psychiatric diseases such as schizophrenia and its neurological causes. EMBO Rep 14: 765-768. [Crossref]

17. Thelen E, Smith L (1994) Dynamic systems approach to the development of cognition and action. Cambridge, MA: MIT Press.

18. Campos JJ, Bertenthal BI, Kermoian R (1992) Early experience and emotional development: the emergence of wariness of heights. Psychol Sci 3: 61-64.

19. Bushnell EW, Boudreau JP (1993) Motor development and the mind: the potential role of motor abilities as a determinant of aspects of perceptual development. Child Dev 64 1005-1021. [Crossref]

20. Lockman JJ, Thelen E (1993) Developmental biodynamics: brain, body, behavior connections. Child Dev 64: 953-959. [Crossref]

21. Shapiro L (2011) Embodied cognition. Routledge Publishing, New York, USA

22. Damasio A (2012) Self Comes to Mind: Constructing the Conscious Brain. Pantheon Books, New York, USA.

23. Hooker CA (2008) Interaction and bio-cognitive order. Synthese 166: 513-54624.

24. Ulanowicz RE (1986) Growth and development: ecosystems phenomenology. Springer-Verlag: Berlin. 
25. Allen M, Friston KJ (2018) From cognitivism to autopoiesis: towards a computational framework for the embodied mind. Synthese 195: 2459-2482. [Crossref]

26. de Vignemont $\mathrm{F}$ (2010) Body schema and body image--pros and cons. Neuropsychologia 48: 669-680. [Crossref]

27. Fourneret P, de Vignemont F, Franck N, Slachevsky A, Dubois B, et al. (2002) Perception of self-generated action in schizophrenia. Cogn Neuropsychiatry 7: 139156. [Crossref]

28. Decety J, Sommerville JA (2003) Shared representations between self and other: a social cognitive neuroscience view. Trends Cogn Sci 7: 527-533. [Crossref]

29. Decety J, Lamm K (2007) The role of the right temporoparietal junction in social interaction: how low-level computational processes contribute to metacognition. Neuroscientist 13: 580-593. [Crossref]
30. Dumas G, Kelso JAS, Nadel J (2014) Tackling the social cognition paradox through multi-scale approaches. Front Psychol 5: 882. [Crossref]

31. Noel JP, Cascio CJ, Wallace MT, Park S (2017) The spatial self in schizophrenia and autism spectrum disorder. Schizophr Res 179: 8-12. [Crossref]

32. Orban GA, Rizzolatti G (2012) An area specifically devoted to tool use in human left inferior parietal lobule. Behav Brain Sci 35: 234. [Crossref]

33. Hopkins J (2016) Free Energy and Virtual Reality in Neuroscience and Psychoanalysis: A Complexity Theory of Dreaming and Mental Disorder. Front Psychol 7: 922. [Crossref]

34. Pobandt T, Knecht V (2014) Free energy of lipid bilayer defects affected by Alzheimer's disease-associated amyloid-B42 monomers. J Phys Chem B 118: 3507-3516. [Crossref]

Copyright: (C2018 Larrivee D. This is an open-access article distributed under the terms of the Creative Commons Attribution License, which permits unrestricted use, distribution, and reproduction in any medium, provided the original author and source are credited. 